\title{
Les tiques des animaux domestiques du Centrafrique
}

\author{
par P. C. MOREL et P. FINELLE
}

Le haut-pays centrafricain est remarquable à plus d'un titre. La dorsale oubangui-charienne de moyenne altitude $(500-1000 \mathrm{~m})$, composée de la pénéplaine ouest, avec le massif de Yadé, et la pénéplaine est, adossée aux massifs du Challa et du Fertit, constitue une séparation entre les bassins du Logone et du Chari au nord (climat soudanien), et le bassin de l'Oubangui au sud (climat subéquatorial). Ce haut-pays est situé à la latitude des savanes guinéennes de l'OuestAfricain, mais présente du fait de son altitude une physionomie propre. Cette échine montagneuse, d'autre part, met en continuité le Moyen-Cameroun ef l'Adamawa avec les massifs du Sudovest du Soudan nilotique et du Congo oriental, qui sont eux-mêmes des contreforts des chaînes et plateaux du haut-pays oriental des lacs. Ainsi par sa face nord le Centrafrique participe à la faune soudanienne, par le sud à la faune équatoriale occidentale, par l'est à la faune d'Afrique orientale. Le centre et l'ouest du pays sont en beaucoup de points comparables au MoyenCameroun et au plateau central de Nigeria.

La nature et la distribution des diverses espèces de tiques du Centrafrique n'ont fait l'objet. jusqu'à présent, que de quelques citations (FIASSON, 1943 ; ROUSSELOT, 1951, 1953 ; MOREL, 1958). Les renseignements que nous publions aujourd'hui, malgré leur petit nombre, permettront de combler quelques lacunes ef de préciser certaines lignes de répartitions d'espèces, à la lumière de ce qu'on sait dans les territoires voisins, où ont eu lieu des prospections plus poussées (Soudan nilotique, Congo oriental, Congo équatorial. Congo occidental (ex MoyenCongo), Cameroun). Cette publication vient à la suite de celles qui intéressent l'Ouest-Africain, le Cameroun et le Tchad. Nous adoptons les

Reçu pour publication : févricr 1961.

Rev. Elev. Méd. vét. Pays trop., 1961, 14, ro 2. mêmes abréviations et conventions que dans les textes auxquels il est fait allusion.

Une carte indique les localités signalées, les courbes isohyètes (d'après FACY, 1950) et les niveau de 500 ef $1000 \mathrm{~m}$. Ceffe carte est commune à la présente publication et à celle de MOREL et GRABER sur les tiques du Tchad.

Fn ce qui concerne les généralités sur la biologie des espèces citées, se reporter à MOREL, 1958.

\section{Amblyomma astrion Dönitz, 1909.}

Localités: Bangui (THEILER et ROBINSON, 1954 ; ROUSSELOT, 1951 : A. cohoerens) ; Bangui, Kouango, Bangassou': bovins ; Bouca : éléphant (IPP).

Primitivement parasite du buffle, A. astrion peut se retrouver chez les bovins qui fréquentent les mêmes parages. II semble remplacer A. splendidum dans les savanes boisées de moyenne altitude du bassin de l'Oubangui et du nord de l'Angola.

\section{Amblyomma nutfalli Dönitz, 1909.}

Localité : Bangui : homme (très nombreuses nymphes dans les narines : (IPP).

Parasite au stade adulte des tortues et varans. en forêt et savanes humides; immatures sur reptiles, oiseaux, petits mammifères; le cas relaté ici semble assez rare.

3. Amblyomma paulopunotatum Neumann, 1899.

Localité : Carnot : potomochère (ROUSSELOT, 1951).

Parasite primitif du potamochère, il a été parfois recueilli sur porc dans les régions forestières.

4. Amblyomma rhinocerotis (de Geer, 1778).

HOOGSTRAAL (1956, p. 249) place en CenI trafrique la localité de Ngourou, d'où NEU- 
MANN (1899: A. aureum) cite cet Amblyomma, spécifique du rhinocéros. Bien qu'il n'y ait pas impossibilité à ce que cette tique ait existé, au moins naguère, en Centrafrique, NEUMANN plaçant Ngourou au «Zanzibar», il est naturel d'assimiler ce nom au Mt Ngourou, près de Mrogoro (Tanganyika), ainsi que l'indique l'étiquette de l'échantillon conservée au MHNP.

\section{Amblyomma splendidum Giebel, 1877.}

Localités: Haute Sanga : buffle (NEUMANN, 1897) ; Nola : buffle (IPP); Berbérati : bœuf (FIASSON, 1943).

Parasite primitif du buffle en forêt équatoriale occidentale et en savane guinéenne, il n'est pas rare de le retrouver sur les bovins domestiques.

\section{Amblyomma tholloni Neumann, 1899.}

Localités : haut Oubangui (NEUMANN, 1899); Nola, Bouca, Badéni, Ndélé (IPP); Birao. Parasite spécifique de l'éléphant.

\section{Amblyomma variegatum (Fabricius, 1794).}

Localités: Bangui, Carnot, Bouar, Bilolo: bovins (ROUSSELOT, 1951) ; Makounda : bœuf (FIASSON, 1943) ; Bouar : bœuf, cheval, porc ; Baboua : bouf; Bouca : éléphant (IPP); Boulakaba : buffle (IPP) ; Fort-Sibut: bceuf (IPP) ; Bayanga (MHNP) ; Nola, Berbérati, Carnot, Paova, Grimari, Bambari, Kouango, Alindao, Ippy, Yalinga, Ouadda, Birao, Ouango, Bangassou, Obo: bovins.

A. variegatum doit donc être vraisemblablement distribué par tout le pays, sauf peut-être dans les montagnes du nord-est ; tout ceci est entièrement en accord avec ce qu'on a observé en Afrique orientale ou dans l'Ouest-Africain. Avec les Boophilus, c'est probablement l'espèce la plus fréquente sur le bétail centrafricain.

\section{Amblycentor circumguttatus (Neumann, 1897)}

Localités: haut Oubangui (Neumann, 1897); Nola, Bouca, Badéni, Ndélé (IPP), Birao.

Parasite spécifique de l'éléphant.

\section{Aponomma flavomaculatum (Lucas, 1846).}

Localité : Ouaga (Bangui) : python (IPP).

Parasite des varans, parfois des pythons, dans les savanes ouest-africaines.
10. Boophilus annulatus (Say, 1821).

Localités : Bangui, Fort-Sibut : bovins (MOREL, 1958) ; Bangui, Yalinga, Ouango : bovins.

Comme dans l'Ouest-Africain, B. annulatus se trouve mêlé en plus ou moins grand nombre aux populations de $B$. decoloratus, au sud de l'isohyète des $1000 \mathrm{~mm}$.

\section{Boophilus decoloratus (Koch, 1844).}

Localités: Bangui, Bilolo: bovins (ROUSSELOT, 1951); Fort-Sibut (IPP); Bangui, Bovar, Ouango, Obo : bovins.

Cf. commentaire à propos de cette espèce très importante comme parasite des bovins dans MOREL (1958).

12. Haemaphysalis leachi leachi (Andouin, 1827).

Localités : Bangui : chien (ROUSSELOT, 1951) Fort-Sibut : chien, Carnot : renard, Bilolo: chien, Fort-Crampel (T. S. DIAS, 1958) ; Bovar: chien; Bambari, Obo: libres; Bouca (IPP).

Parasite des carnivores sauvages, qui se retrouve fréquemment sur les domestiques, $H$. 1. leachi doit être présent sous toutes les latitudes du Centrafrique.

13. Haemaphysalis leachi muhsamae T. S. Dias, 1954.

Localités : Bilolo: genette (T. S. DIAS, 1958).

Parasite des petits carnivores Mustélidés ef Herpestidés, de même distribution que la forme typique.

14. Haemaphysalis parmata Neumann, 1904.

Localités : Carnot : chien (ROUSSELOT, 1953 ; correction probable de ROUSSELOT, 1951 : H. aciculifer) ; Bilolo, Nola : Cephalophus, sp. (ROUSSELOT, 1951, 1953); Zendi : chien (IPP).; Bouar : chien, chat.

Parasite des mammifères des zones de forêt équatorial et des savanes boisées occidentale, surtout des ruminants, à un moindre degré des porcins et carnivores, dans des zones recevant au moins $1250: \mathrm{mm}$ de pluies annuelles. En Centrafrique l'espèce semble bien établie dans le sud-ovest; sa: présence est probable dans la galerie forestière de l'Oubangui et du Mbomou. 


\section{Hyalomma impressum Koch, 1844.}

Localité : Bangui (ROUSSELOT, 1951, 1953).

Espèce des savanes soudaniennes; dans les zones plus humides ou plus boisées on n'en trouve plus que quelques populations éparses, ou des introductions artificielles dues à la transhumance. Dans l'Ouest-Africain $H$. impressum (ainsi que $H$. rufipes) cesse d'être numériquement important au sud de l'isohyète des $1000 \mathrm{~mm}$.

\section{Hyalomma rufipes Koch, 1844.}

Localitćs : Bangui (ROUSSELOT, 1951, 1953). Bangui, Bovar, Grimari, Birao: bovins (quelques exemplaires); Birao ( $n n)$ : pintade.

Cf. remarques concernant l'espèce précédente.

\section{Hyalomma truncatum Koch, 1844.}

Localités : Bangui (ROUSSELOT, 1951, 1953); Fort-Bousseaux (FIASSON, 1943: H. aegyptium); Bossangoa: buffle (IPP) ; Bouar, Bocaranga, Baboua, Bangui, Grimari, Kouango : bovins.

Comme dans I'Ouest-Africain cette espèce, à la différence des deux précédentes, s'accommode d'une plus forte pluviométrie quoique son maximum numérique se situe en savane soudanienne.

\section{Rhipicephalus appendiculotus Neumann, 1901}

Localités: Bangui : bovin (ROUSSELOT, 1951); Obo : bovin (1 m, 2 ff).

La présence de ce rhipicéphale en deux points de la frontière sud du Centrafrique, sur les rives de l'Oubangui et du Mbomou, se comprend en considérant la distribution au Congo oriental et au Congo équatorial. Le risque d'introduction de populations plus ou moins importantes de cette espèce, et leur établissement, constituent une grave menace pour le cheptel centrafricain, surtout dans l'est, où les conditions d'altitude et de pluviométrie présentent les caractéristiques favorables au développement de la tique, telles qu'on les observe en Afrique orientale. La constatation de foyers de théilériose bovine de la côte orientale ne représenterait donc pas un phénomène surprenant. explicable par l'extension plus ou moins rapide du vecteur (pour des causes diverses, naturelles ou artificielles), ou encore l'introduction de troupeaux non prémunis dans des régions où les bovins originaires auraient masqué la maladie en raison d'une certaine prémunition naturelle. Nous indiquons sur la carte les emplacements où la présence de $R h$. appendiculatus a été reconnue, dans les pays voisins du Centrafrique.

\section{Rhipicephalus ourantiocus Neumann, 1906.}

Nous pensons que c'est sous ce nom qu'il faut ranger les $R h$. longicoxatus de ROUSSELOT $(1951,1953)$ pour les raisons suivantes. Al'époque de ces publications les exemplaires originaux de longicoxatus étaient perdus. HOOGSTRAAL a réidentifié et redécrit l'espèce sur des tiques de Côte des Somalis, Somaliland, Kenya, Soudarı nilotique (1953, 1956). Biologiquement et morphologiquement le véritable longicoxatus est très caractéristique, et les détails que donne ROUSSELOT sur les tiques qu'il appelle de ce nom ne concordent pas avec les textes d'HOOGSTRAAL. II est done des plus vraisemblable que ROUSSELOT a observé une espèce remarquable par ses hanches I, sans qu'il faille pour ce seul foit l'assimiler à longicoxatus. Maltheureusement à la même époque l'attention n'était pas attirée sur la particulière morphologie coxale de $R h$. aurantiacus. $\angle U M P T$ (1943) n'en parle pas. Nous l'avons trouvée constante sur des' exemplaires du Cameroun (MOREL ef MOUCHET, 1958), ainsi que sur les types de NEUMANN (Toulouse, no 1444, Liberia). ROUSSELOT dił lui-même (1953, p. 80, note) que son espèce est très proche de ziemanni; son dessin du mâle est schématique, mais se rapproche plus d'aurantiocus que des dessins d'HOOGSTRAAL pour longicoxatus ; le dessin de la femelle est entièrement caractéristique d'aurontıacus, et ne peut se comparer à la femelle de longicoxatus.

Les exemplaires de ROUSSELOT proviennent de Carnot sur potamochère (ainsi que de Kellé, Congo). Hôte et localités s'accordent avec toutes nos références concernant $R h$. aurantiacus du bloc forestier guinéo-congolais.

\section{Rhipicephalus cuspidatus Neumann, 1907.}

Localité : Ndélé : phocochère (IPP).

Parasite fréquent de l'oryctérope ef du phacochère, dans les savanes sahéliennes et soudaniennes. 
CARTE 1. - TCHAD ET CENTRAFRIQUE

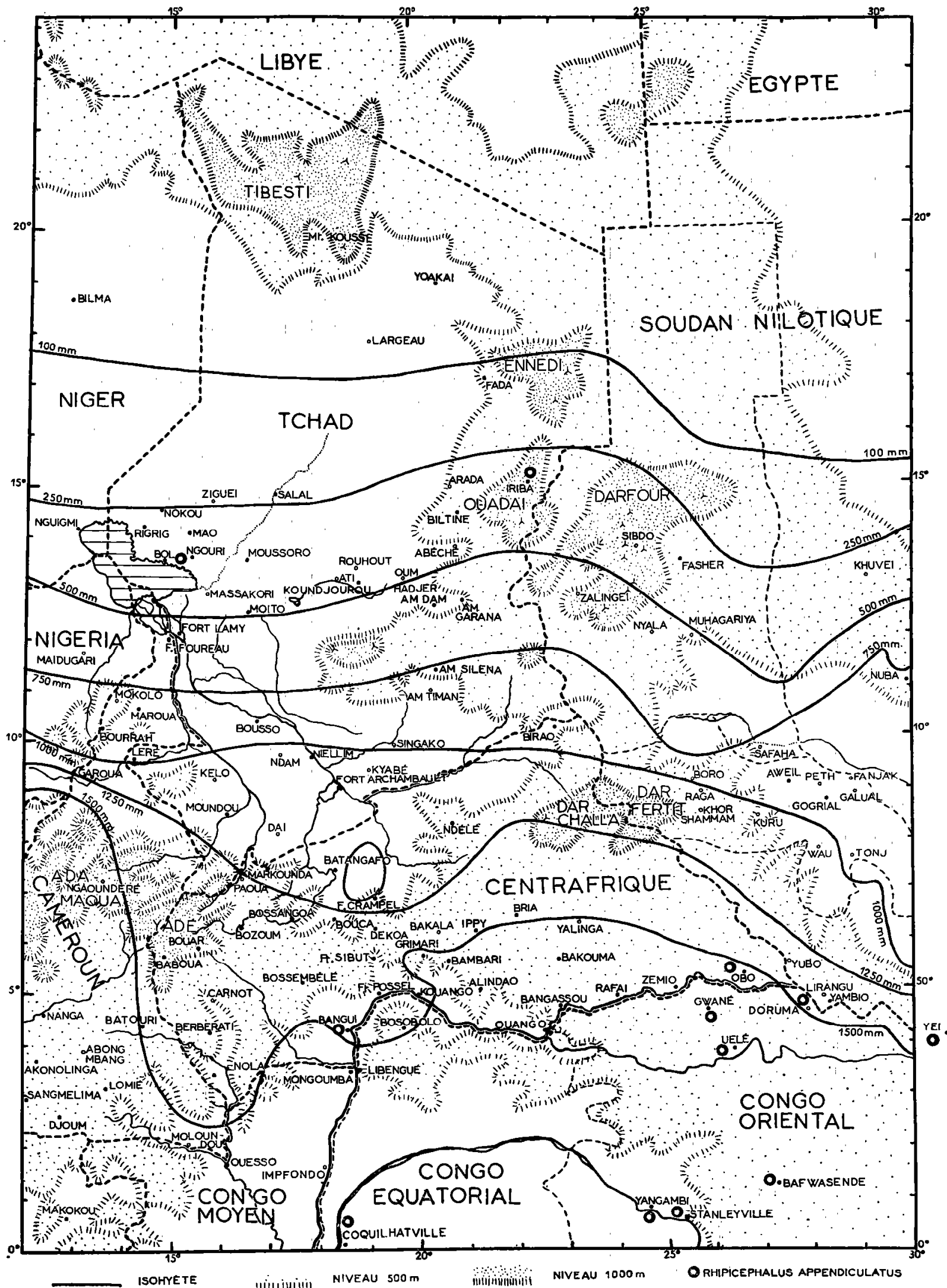




\section{Rhipicepholus evertsi Neumann, 1897.}

Localités: Bangui : bovins (ROUSSELOT, 1951,1953) ; Fort-Sibut : cheval (IPP) ; Obo : bovin.

\section{Rhipicephalus longus Neumann, 1907.}

Localitćs : Bangui : bœuf, mouton (ROUSSELOT, 1951, 1953): Nola : buffle (IPP): Bossangoa, Bouca : buffle (IPP); Bovar, Baboua, Ippy, Bangui, Ouango, Yalinga, Bangassou, Obo : bovins ; Birao: éléphont.

Espèce typique des savanes humides ef de la forêt équatoriale occidentale, et dont l'hôte spécifique originel cst le buffle. Dans l'OuestAfricain, on ne l'a pas encore retrouvé sur les bovins domestiques (de même qu'A. splendidum, autre tique du buffle, n'a été que rarement recueilli sur le bœuf), alors qu'en Centrafrique, Cameroun, bassin de l'Oubangui et du Congo, Soudan nilotique, etc..., il parasite courammentle bétail, au même titre que $R h$. simus senegalensis ; la raison de ce double comportement à l'ovest et à l'est du plateau central du Nigeria et du massif camerounais est peu claire; il est possible que le facteur d'altitude favorise le développement numérique de $R h$. longus; il est vraisemblable d'autre part de penser que les facteurs humains (extension des cultures, développement de la chasse) ont modifié plus ou moins les conditions naturelles dans l'Ouest-Africain, isolant ainsi la faune des ruminants sauvages dans des zones moins favorables à l'homme; le buffle dans ces conditions se trouverait confiné dans des régions où l'élevage des bovins n'est pas pratiqué, de sorte qu'il n'y aurait pas interférence entre le parasitisme des Bovidés sauvages et domestiques (Rh. longus est présent au Liberia : NEUMANN. 1907 ; au Togo : ZUMPT, 1942 ; en Côte-d'Ivoire lagune d'Assagny et Toupé (Kakpin), sur buffle ef sur herbes).

\section{Rhipicephalus lunulatus Neumann, 1907.}

Localités: Baboua : herbes et Fort-Sibut : 1 porc, chien (MOREL, 1958); Yalinga : bœuf.

Espèce rencontrée en petit nombre, mêlée aux populations de $R h$. simus senegalensis ou $R h$. simus simus.
24. Rhipicephalus planus complanatus Neumann, 1910.

Localité : Bangui : 3 mâles sur porc.

Parasite typique du potamochère en forêt équatoriale occidentale, où il se retrouve parfois, comme c'est le cas ici, sur les porcins domestiques.

\section{Rhipicephalus sanguineus (Latreille, 1806).}

Localités: Nola, Bilolo, Carnot : chien (ROUSSELOT, 1951, 1953) ; Zendi : chien (IPP) ; Fort-Sibut: âne, porc, mouton, chien, chat, rot roussard (Arvicanthis niloticus : $2 \mathrm{nn}$ ) (iPP) ; Fort-Crampel (MHNP); Bangui : cnien, cheval (IPP) : Baboua, lppy : herbes ; Bouar, Bambari, Kovango : chien. ; Carnot: renord, chien (IPP).

Espèce non homogène, d'une grande variabilité morphologique ou biologique suivant les hôtes ou la zone d'origine. En ce qui concerne l'Afrique éthiopienne, on peut au moins distinguer une «race» domestique, dont tous les stades évolvent sur le chien, et une «race» sauvage, dont les larves et nymphes évoluent sur les Rongeurs Myomorphes el les adultes sur les herbivores domestiques et sauvages (biologie très comparable à celles des Rhipicephalus du groupe simus ou capensis). II est d'autant plus regrettable que la nature réelle du'complexe songuineus ne soit pas éclairci, qu'on a montré que certaines populations de ce complexe sont vecirices, naturellement ou expérimentalement, de la plupart des Sporozoaires des herbivores ef carnivores domestiques, ainsi que de diverses affections à rickettsies, bactéries ou ultra-virus.

\section{Rhipicephalus simus simus Koch, 1844.}

Localités: Bangui : bœuf, phacochère (ROUSSELOT, 1951, 1953); Fort-Sibut: porc (IPP) ; Baboua : herbes.

\section{Rhipicephalus simus senegalensis Koch, 1844.}

Localités: Bangui : bovins (ROUSSELOT, 1951, 1953) ; Fort-Sibut: bovin, mouton, chèvre (IPP) ; ippy, Ouango, Obo : bovins ; Bossangoa : buffle (IPP).

Les deux sous-espèces de simus se distinguent dans l'Ouest-Africain, par leurs distributions, en relation avec des exigences hygrométriques 
différentes. Le simus typique est une tique des savanes soudaniennes, présente en quelques îlots au sud de l'isohyète des $1000 \mathrm{~mm}$; $R h$. simus senegalensis apparaît plus méridional, surtout abondant au delà de $1.200 \mathrm{~mm}$, donc dans les savanes humides de type guinéen ; il en est probablement de même dans les savanes oubangui-chariennes, avec cœxistence du simus simus dans la savane ovest.

\section{Rhipicephalus simpsoni Nuttall, 1910.}

Les Rh. simus senegalensis de ROUSSELOT (1951, 1953) recueillis à Bangui sur aulacode (Thryonomys swinderianus) appartiennent vraisemblablement à l'espèce simpsoni, qui est précisément spécifique de ce rongeur, très commun dans les savanes humides ef en forêf autour des plantations.

29. Ixodes cumulatimpunctatus Schulze, 1943 (= 1. pseudorasus Arthur et Burrow, 1957).

Localité : Nulu: Cephalophus sp. (ROUSSELOT 1951, 1953) : $1 \mathrm{~m}, 1$. rasus ; correction établie après examen du matériel de ROUSSELOT déposé au Service de l'Elevage à Brazzaville).

Espèce fréquente sur les mammifères de la zone équatoriale (forêł guinéo-congolaise, Ouganda, Kenya, Tanganyika, Rhodésies).

\section{Ixodes rageaui Arthur, 1958.}

Localité : Nola : Cercopithecus cephus (ROUSSELOT, $1953: 1 \mathrm{~m}$ l. ugandanus).

Tique spécifique des cercopithèques du bloc forestier congolais.

\section{CONCLUSIONS}

Les tiques du Centrafrique s'intègrent naturellement dans la faune des savanes de moyenne altitude qui vont du Moyen-Cameroun au sudovest du Soudan nilotique (Bahr el Ghazal, Equatoria ovest). Comme dans les savanes guinéennes de l'Ouest-Africain, les principaux parasites du bétail, numériquement et du point de vue des agents pathogènes transmis, sont $A$. variegatum, $B$. decoloratus, $B$. annulatus, puis à un moindre degré $R h$. simus senegalensis et $R h$. sanguineus (race sauvage) ; à la différence de l'Ouest-Africain, sur cette dorsale camerounonilotique $R h$. longus apparaît avec une importance égale ou supérieure à celles des autres rhipicéphales.' A ces éléments s'ajoutent des prolongements de la faune des massifs et hauts plateaux d'Afrique orientale, représentés par $R h$. appendiculatus, dont on ignore l'importance exacte dans l'est du Centrafrique, ainsi que sa signification en rapport avec l'existence de la theilériose bovine à Th. parva.

II n'y a pas eu de récolte d'Argas de volailles. En'se fondant sur les données d'Afrique occidentale, on peut supposer qu'A. persicus est présent dans le nord, et que dans le reste du territoire, hormis la forêt, l'espèce en cause est A. hermanni (existant en Guinée, Côte-d'Ivoire, Haute-Volta, Dahomey).

Institut d'élevage et de médecine vétérinaire des pays tropicaux:

Laboratoire national de Centre de recherches l'élevage

«Georges Curasson 》, trypanosomiases animales Dakar-Hann Sénégal. Bouar (Rép. Centrafricaine)

\section{RÉSUMÉ}

Les auteurs donnent un aperçu d'ensemble sur les tiques des vertébrés domestiques ef sauvages du Centrafrique, en comparant ces données, personnelles ou bibliographiques, à celles qui existent pour les territoires voisins. La présence de Rhipicephalus appendiculatus est indiquée, avec les conséquences touchant l'existence corrélative de Theilerio parva.

\section{SUMMARY}

\section{Ticks of domestic animals in the Central African Republic}

The authors give an over-all picture of the ticks in both domestic and feral vertebrates in the Central African Republic, and compare these with personal and published notes on those of neighbouring tcrritorics. The presencc of Rhipicephalus appendiculatus is recorded and reference is made to the consequence of the possible co-existence of Theileria parva. 


\section{RESUMEN}

\section{Las garrapatas de los animales domésticos del Africa Central}

Los autores dan un resumen sobre las garrapatas de los vertebrados domésticos y salvajes del Africa Central comparando estos datos personales o bibliográficos, a aquellos que existen para los territorios vecinos. Se indica la presencia de Rhipicephalus appendiculatus con las consecuencias que corresponden a la existencia correlativa de Theileria parva.

\section{BIBLIOGRAPHIE}

DIAS (J. A. Travassos Santos). - Notes on various ticks (Acarina, lxodidae) in collection at some entomological institutes in Paris and London. An. Inst. Med. trop., 1958, 15 (2) : 459-653.

FACY (L.). - Météorologie in Guernier (E.). Afrique-Equatoriale Française. Paris (Encycl. marit. colon., 590 pp.), $1950: 159-72$.

FIASSON (R.). - Contribution à l'étude des arthropodes vulnérants du Moyen-Congo. Rev. Sci. méd. Afr. franç. libre, 1943, 2 (2) : I 125-51.

HOOGSTRAAL (H.). - African Ixodoidea. I. Ticks of the Sudan. Research Report NM 005 050. 29.07, U. S. Govt. Print. Office. 1956-0-390 800, 1101 pp.

MOREL ( $P . \quad C$.$) . - Les tiques des animaux$ domestiques de l'Afrique-Occidentale Française. Rev. Elev. Méd. vét. Pays trop., 1958, II (2) : 153-89.

MOREL ( $P, C$.) ef MOUCHET (J.). - Les tiques du Cameroun (Ixodidae el Argasidae). Ann. Parasit. hum. comp., 1958, 33 (1-2) : 69-111.

PIERQUIN (L.) et NIEMEGEERS (K.). - Répertoire ef distribution géographique des tiques au Congo belge et au Ruanda-Urundi. Bull. ogric. Congo belge, 1957, 48 (5) : 1177-224.

RAGEAU (J.). - Ixodidés du Cameroun. Bull. Soc. Path. exot., 1951, 44 (7-8) : 441-6.

RAGEAU (J.). - Note complémentaire sur les Ixodidae du Cameroun. Bull. Soc. Path. exot., 1953, $46(6): 1090-8$.

ROUSSELOT (R.). -... Ixodes de l'Afrique noire. Bull. Soc. Path. exot. 1951, 44 (5-6) : 307-9.

ROUSSELOT (R.). - Notes de parasitologie tropicale. II. Ixodes. Paris (Vigot édit.) 1953: 1-135.

THEILER (G.) ef ROBINSON (B. N.). - Tick Survey. VIII. Checklists of ticks recorded from the Congo and Ruanda-Urundi, from Angola and from Northern Rhodesia. Onderst. J. vet. Res., 1954, 26 (3) : 447-61.

ZUMPT (F.). - Vorstudie zu einer Revision der Gattung Rhipicepholus Koch. V. Zur Kennt$n$ is afrikanischer Rhipicephalus Arten. Zeitschr. Parositenk., 1942 b, 12 (4) : 479-500.

ZUMPT (F.). - Vorstudie zu einer Revision der Gattung Rhipicephalus Kock. VIII. Rhipicephalus aurantiacus Neum. und verwandte Arten. Zeitschr. Porositenk., 1943 b, 13 (1) : 102-17. 